\title{
Joëlle Ducos and Christopher Lucken (eds.), Richard de Fournival et les sciences au XIII siècle. Micrologus Library 88, Firenze, SISMEL, 2018, vi + 443 pp., ISBN: 97888845 08430. Cloth €68
}

\author{
Reseñado por BÉATRICE DELAURENTI \\ EHESS, Centre de Recherches Historiques, Paris, FR \\ beatrice.delaurenti@ehess.fr
}

Parfois, les poètes sont aussi médecins, mathématiciens ou astrologues. Leur œuvre se trouve alors scindée en deux, étudiée d'un côté par les historiens de la littérature, mais seulement partiellement, méconnue de l'autre côté par les historiens des sciences. Richard de Fournival (1201-1260) est un exemple de cette difficulté et des angles morts qu'elle contribue à créer. Connu aujourd'hui pour ses poèmes amoureux, ses chansons religieuses et les traités sur l'amour qui lui sont attribués, cet auteur peut pourtant aussi être abordé sous une autre perspective, à partir de sa production scientifique. Le volume dirigé par Joelle Ducos et Christopher Lucken, Richard de Fournival et les sciences, entend rétablir l'équilibre en étudiant deux types d'écrits : d'une part la Biblionomia, cette bibliothèque idéale, au moins en partie constituée, dont Richard de Fournival a décrit les différentes section à la manière d'un jardin rassemblant tout le savoir de manière organisé ; d'autres part divers écrits dont l'authenticité est incertaine et sur lesquels le volume revient à nouveaux frais - De arte alchemica, Nativitas, De vetula, Speculum astronomie -, faisant le point sur les arguments favorables ou opposés à leur attribution à Richard de Fournival.

L'ouvrage s'ouvre par une introduction de Christopher Lucken qui détaille les éléments connus de la vie de Richard : sa formation à la faculté des arts de l'université de Paris, puis sans doute dans une faculté de médecine européenne (Paris, Montpellier ou Salerne) ; sa carrière ecclésiastique achevée comme chancelier de la cathédrale d'Amiens ; son séjour à la cour pontificale ; l'importance de la ville d'Amiens dans son parcours, comme lieu d'une articulation fine entre sciences et techniques. En miroir, la conclusion de Joëlle Ducos complète le portrait par d'autres éléments : elle souligne le bilinguisme latin-français de Richard de Fournival, son intérêt pour les savoirs concrets plutôt qu'abstraits, la liberté d'écriture que permet l'emploi des langues vernaculaires, autant d'aspects qui caractérisent la culture savante de la première moitié du XIII ${ }^{\mathrm{e}}$ siècle dont Richard, « figure emblématique du désir de savoir » (p. 22), s'est fait le « passeur » (p. 407).

Cette entrée en matière et cet épilogue décrivent le contexte intellectuel dans lequel s'est déployé l'intérêt de Richard de Fournival pour les sciences. Entre les deux, les treize études réunies dans le volume dressent un riche panorama des activités scientifiques du personnage, montrant son importance et révélant les zones d'ombre qui demeurent autour de sa production intellectuelle. Trois types d'éclairages sont proposés. Un premier groupe d'articles étudie dans son ensemble le projet intellectuel de la fameuse Biblionomia. 
Christopher Lucken en décrit les différentes sections. Jean-Marc Mandosio examine les principes de classification du savoir qui fondent le regroupement des ouvrages, mettant en valeur les choix de Richard et ses omissions. Isabelle Draelants compare les ouvrages listés dans la Biblionomia avec les sources utilisées par les encyclopédistes de la même période.

D'autres contributions se centrent sur une section particulière de la bibliothèque-jardin : médecine, mathématiques et secrets sont ainsi passés en revue. En médecine, l'étude de Monica Green met en valeur le rôle pionnier de Richard. La Biblionomia comprend des livres de médecine rares du corpus de textes traduits du grec ou de l'arabe au XI et au XII siècle; dans certains cas, il s'agit même de la première attestation de ces textes en traduction latine. Richard aurait aussi composé lui-même certains recueils manuscrits, assemblant des textes dans un but pédagogique. Ces deux gestes « herculéens » (p. 181), acquérir et assembler, ont eu une importance capitale pour la transformation de la médecine savante au XIII siècle. D'autres articles mettent l'accent sur les spécialités médicales pour lesquelles la Biblionomia comprend quelques témoins. Martina Giese s'intéresse à la médecine vétérinaire, identifiant et datant les deux opus mentionnés dans la Biblionomia, réfléchissant aussi à la position de Richard à l'égard de ces textes : pourrait-il en avoir été le dédicataire? Laurence Moulinier enquête sur les sept textes d'uroscopie de la Biblionomia, constatant avec perplexité qu'ils sont peu nombreux, même s'il s'agit de textes importants : Richard de Fournival ne manifeste pas un intérêt marqué pour la science des urines, alors qu'il semble avoir lui-même été une autorité en la matière. Mais ses écrits sur les urines, malheureusement, sont perdus.

En mathématiques, la Biblionomia aménage une place notable à l'arithmétique et à la géométrie, sur lesquelles deux contributions font le point. Marc Moyon dresse un panorama des livres énumérés dans ce domaine, parmi lesquels plusieurs écrits de Jordanus de Nemore. Il formule l'hypothèse d'une relation de maitre à élève entre Richard et Jordanus et constate par contraste l'absence fort surprenante, dans la Biblionomia, des écrits de Fibonacci. Laure Miolo étudie la réception des livres de mathématiques de Richard de Fournival. Une partie des ouvrages composant la Biblionomia fut en effet léguée par Gérard d'Abbeville au collège de la Sorbonne après la mort de Richard, en 1272. Ce leg a eu des effets de long terme, contribuant à transformer la bibliothèque de la Sorbonne en « vecteur de la renovatio scientifique du XIV ${ }^{e}$ siècle » (p. 175). La section réservée aux livres secrets, enfin, est explorée par Nicolas Weill-Parot, qui compare l'économie du secret de la Biblionomia et celle d'une autre fameuse liste d'ouvrages, anonyme celle-ci, le Speculum astronomie. L'examen révèle deux manières différentes de penser la relation entre connaissance et secret : d'un côté, le Speculum développe une « philosophie de l'occulte » fondée sur l'idée qu'il existe dans la nature une part indépassable d'inconnu ; de l'autre côté, Richard de Fournival défend une " philosophie du secret » (p. 336) portée par une confiance dans la capacité humaine à découvrir, les uns après les autres, tous les secrets du monde. 
Un troisième groupe d'articles porte sur d'autres textes écrits par, ou attribués à Richard de Fournival. Antoine Calvet présente le De arte alchemia en concluant qu'il est vraisemblablement authentique. Il souligne la forme inhabituelle du traité, qui se présente comme l'exposition didactique d'une expérience de transmutation relatée à la première personne, sans théorie ni citations d'autorités. Une édition et une traduction française du texte sont annexées à l'article. La Nativitas est également jugée authentique par Jean-Patrice Boudet et Christopher Lucken, qui font le point sur l'intérêt de Richard pour l'astrologie et sur la méthode de calcul employée dans cet horoscope. Quant au De vetula, poème astronomique et astrologique faussement attribué à Ovide, Cecilia Panti propose de le dater entre 1240 et 1260, mais reste réticente sur une possible attribution à Richard de Fournival. Elle met par ailleurs en lumière la circulation de ce texte en France et en Angleterre jusqu'au XV $\mathrm{X}^{\mathrm{e}}$ siècle, en lien avec les écrits de Roger Bacon. Marie-Madeleine Huchet, de son côté, étudie la place des sciences du quadrivium dans le De vetula et la position prépondérante de l'arithmétique et de la science des astres. Le Speculum astronomie, enfin, est examiné par Charles Burnett dans un court article qui revient sur une question déjà longuement débattue, celle de son auteur. L'identité de celui-ci reste inconnue, malgré les divers noms déjà suggérés - Campanus de Novare, Roger Bacon, Albert le Grand ? La possibilité d'attribuer le Speculum astronomie à Richard de Fournival avait été soutenue en 2000 par Bruno Roy sans rencontrer beaucoup d'écho. Reprenant les différents éléments du débat, Burnett constate qu'une telle attribution serait, en définitive, la plus logique, même si aucun argument n'est décisif et qu'il faut se contenter, pour l'heure, de simples présomptions. Le débat reste ouvert : dans leur propre article, Jean-Patrice Boudet et Christopher Lucken concluent en sens inverse, considérant que l'écart de vocabulaire entre la Nativitas de Richard de Fournival et le Speculum astronomie ne plaide pas en faveur d'une telle attribution.

De la médecine aux mathématiques, de l'astrologie à l'alchimie, les contributions de cet ouvrage restituent ainsi à Richard de Fournival toute l'épaisseur et la diversité de ses intérêts intellectuels. Elles mobilisent des domaines de compétences variés autour d'un personnage singulier et fascinant. Chaque contribution s'adresse aux spécialistes d'un champ, poursuivant des débats parfois techniques, mais le propos d'ensemble est lisible par tous et éclairant, y compris pour les non-spécialistes. L'effet obtenu est saisissant: l'ouvrage montre quels bénéfices l'étude de la pensée médiévale retire du croisement des perspectives et des éruditions. À l'issue de cette lecture, Richard de Fournival a gagné en densité et en complexité, sans pour autant perdre son caractère mystérieux. 